\title{
Post-challenge hyperglycaemia is associated with premature death and macrovascular complications
}

\author{
Q. Qiao', ${ }^{1,}$, J. Tuomilehto ${ }^{2,3}$, K. Borch-Johnsen ${ }^{4}$ \\ ${ }^{1}$ Mannerheimintie 166, Helsinki, Finland \\ 2 Diabetes and Genetic Epidemiology Unit, National Public Health Institute, Helsinki, Finland \\ ${ }^{3}$ Department of Public Health, University of Helsinki, Finland \\ ${ }^{4}$ Steno Diabetes Center, Gentofte, Denmark
}

\begin{abstract}
Unravelling the precise association between cardiovascular disease and Type 2 (non-insulin-dependent) diabetes mellitus has proved problematic, largely as a result of the confusing variety of diagnostic criteria and methodologies used in previous studies of disease-associated risk. More recently, improved standardization and continuing refinements to risk analyses have begun to clarify the relative importance of individual risk factors for macrovascular complications and progression to cardiovascular disease. The evidence accumulated from several large-scale epidemiological and intervention studies strongly indicates that hyperglycaemia and specifically the post-load hyperglycaemia resulting from postprandial glucose excursions is the main factor associated with increasing the risks of morbidity and mortality in Type 2 diabetes. The DECODE study has de-emphasized the importance of fasting glucose, in contrast to post-load hyperglycaemia, as the principal measurable determi-
\end{abstract}

nant of exposure to the risk of cardiovascular disease in Type 2 diabetes. Similarly, the Diabetes Intervention Study identified mealtime hyperglycaemia as an independent risk factor for myocardial infarction and all-cause mortality, while several other studies have also shown that such mortality is better predicted from post-challenge measurement criteria rather than from fasting-glucose criteria. It is likely that postprandial hyperglycaemia is an independent risk factor for atherosclerosis and is also exerting an atherogenic effect indirectly through the clustering of a number of risk factors for atherosclerosis. Whether the relation between post-load hyperglycaemia and the risk of cardiovascular events is causal is still not known. [Diabetologia (2003) 46[Suppl1]:M17-M21]

Keywords Type 2 diabetes, Post-challenge hyperglycaemia, Coronary heart disease, Cardiovascular disease, Impaired glucose tolerance, Impaired fasting glycaemia, 2-hour glucose concentration.
It has long been established that overt diabetes is associated with a markedly increased risk of cardiovascular disease (CVD) and that this excess risk is not ex-

Received: 17 April 2001 / Revised: 26 July 2002

Published online: 7 November 2002

(C) Springer-Verlag 2002

Corresponding author: Q. Qiao, Mannerheimintie 166, 00300 Helsinki, Finland, E-mail: qing.qiao@ktl.fi

Abbreviations: CVD, Cardiovascular disease; CHD, coronary heart disease; FPG, fasting plasma glucose; MI, myocardial infarction; IMT, intima-media thickness; DECODE, Diabetes Epidemiology: Collaborative analysis of Diagnostic criteria in Europe; DIS, Diabetes Intervention Study; NDDG, National Diabetes Data Group; WHO, World Health Organization. plained by the increase of other conventional cardiovascular risk factors. Hyperglycaemia itself could possibly be responsible for the increased cardiovascular risk and glucose concentration might be a cardiovascular risk factor across its entire range, including the non-diabetic range. The issue of whether asymptomatic hyperglycaemia is likewise a risk factor for CVD has been investigated for at least two decades. In 1979, a series of papers from the International Collaborative Group [1] did not show consistent evidence for a threshold or graded association between asymptomatic hyperglycaemia and coronary heart disease (CHD). In 1985, 13 cohort studies on post-challenge glucose and CHD were reviewed [2]. Five studies 
found a positive association between post-challenge glucose and CHD that remained statistically significant after multivariate analysis. Two studies showed a positive association that was not persistent after adjusting for covariates or no multivariate analysis was done and the remaining six did not show an association even before other risk factors were considered. Based on the review, the author concluded that glucose intolerance is not an established risk factor, unlike serum lipids, blood pressure and smoking.

Over the past 20 years, several developments have contributed to a sharper delineation of these issues. One of the most important facts is that the prevalence of diabetes has increased markedly. This major development resulted from the near-universal adoption of the National Diabetes Data Group (NDDG) [3] and World Health Organization (WHO) $[4,5]$ criteria for diagnosing diabetes. Before such criteria were put into effect enormous variations existed in diagnostic cutoff values both for fasting glucose and after glucose loading. The amount of the glucose load varied between $50 \mathrm{~g}$ and $100 \mathrm{~g}$ or was related to body weight. The differences in glucose assay methods, glucose load and the time after loading, follow-up time and the populations studied probably contributed to the inconsistent observations from the early studies.

Recently, increasing evidence indicates that postload hyperglycaemia could be of greater importance. The 23-year follow-up of the Honolulu Heart Program suggested that an increasing risk gradient existed between CHD and a glucose concentration $1 \mathrm{~h}$ after a 50 g glucose load [6]. In the Funagata diabetes study, analysis of survival rates concluded that IGT, but not IFG, was a risk factor for CVD [7]. In the Hoorn study on older patients without diabetes, an association was found between a 2 -h post-challenge glucose load and predicted mortality which proved to be a better indicator than fasting glucose or $\mathrm{HbA}_{1 \mathrm{c}}$ [8]. A 20-year follow-up of non-diabetic men using data from the Whitehall, Paris Prospective and Helsinki Policemen studies [9] indicated that men in the upper $2.5 \%$ of the 2-h glucose distribution had a higher risk of CHD death compared with those in the lower $80 \%$, with an age-adjusted hazard ratio of 1.8 (95\% CI 1.4-2.4). Based on longitudinal studies in Mauritius, Fiji and Nauru, it has been shown that isolated postchallenge hyperglycaemia $(\geq 11.1 \mathrm{mmol} / \mathrm{l})$ with a normal FPG $(<7.0 \mathrm{mmol} / \mathrm{l})$ doubles the risk of mortality [10] (Fig. 1). A recent systematic overview and metaregression analysis of epidemiological studies found that a progressive association between glucose concentrations and cardiovascular risk extends below the diabetic threshold in non-diabetic men [11].

In older women from California, isolated post-challenge hyperglycaemia more than doubled cardiovascular mortality [12]. This is important because post-challenge glucose concentrations increase to a much greater extent with advancing age than fasting glucose
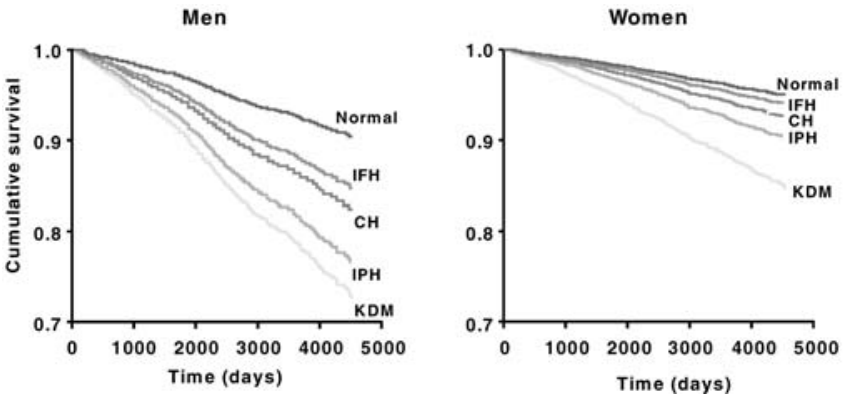

Fig. 1. Adjusted survival (Cox proportional hazard analyses for all-cause mortality adjusted for age and ethnicity), according to diabetes categories for males, and females. Isolated fasting hyperglycemia (IFH): fasting plasma glucose (FPG) $\geq 7.0 \mathrm{mmol} / \mathrm{l}$ and 2-h plasma glucose (2h-PG) $<11.1 \mathrm{mmol} / \mathrm{l}$; isolated post-challenge hyperglycaemia (IPH): $\mathrm{FPG}<7.0 \mathrm{mmol} / \mathrm{l}$ and $2 \mathrm{~h}-\mathrm{PG} \geq 11.1 \mathrm{mmol} / \mathrm{l}$; combined hyperglycaemia $(\mathrm{CH})$ : FPG $\geq 7.0 \mathrm{mmol} / \mathrm{l}$ and $2 \mathrm{hPG} \geq 11.1 \mathrm{mmol} / \mathrm{l}$; known diabetes (KDM) [10]

[12]. In the DECODE population, the prevalence of isolated post-load hyperglycaemia (i.e. 2-h plasma glucose $\geq 11.1 \mathrm{mmol} / \mathrm{l}$ and $\mathrm{FPG}<7.0 \mathrm{mmol} / \mathrm{l}$ ) was $0.7 \%$ in people who were 49 years of age or younger and $4.6 \%$ in those older than 70 years. In contrast, the prevalence of isolated fasting hyperglycaemia, (i.e. 2-h plasma glucose $<11.1 \mathrm{mmol} / \mathrm{l}$ and FPG $\geq 7.0 \mathrm{mmol} / \mathrm{l}$ ) in these two age groups was $1.3 \%$ and $2.3 \%$, respectively (unpublished DECODE data). A sharp increase in post-load hyperglycaemia was observed with increasing age. Fasting glucose concentrations in this respect can be compared with diastolic blood pressure that does not rise with age, whereas systolic blood pressure does and is a stronger predictor of cardiovascular complications [13]. With conclusive evidence, the guidelines for the treatment of hypertension have been modified to emphasise the importance of systolic blood pressure [14]. The need for similar assessment regarding mealtime versus fasting glucose and for evaluating the possible benefit of treatments focused on postprandial hyperglycemia is required, especially in the elderly population.

More recently, analyses of the DECODE database, using multivariate Cox proportional hazard analyses, showed that abnormalities in 2-h glucose were better predictors of mortality from all-cause mortality and from cardiovascular and non-cardiovascular disease than fasting glucose alone $[15,16]$. A high 2-h glucose concentration was associated with an increased risk of death, independent of the concentration of fasting blood glucose, whereas mortality associated with the fasting glucose concentration depended on the concentration of 2-h glucose [16] (Table 1). Adding 2-h glucose to a model based on fasting glucose improved the prediction (all $p$ values $<0.05$, except for stroke). Conversely, fasting glucose did not add any information once 2-h glucose concentration was in the model (all $p>0.10$ ) [16]. The largest absolute high number of deaths was ob- 
Table 1. Adjusted hazard ratios (95\% CI) for death from CVD, CHD, stroke and all-cause mortality when both the fasting and the 2-h glucose classes were in the same model. Subjects not known as diabetic in the DECODE study

\begin{tabular}{|c|c|c|c|c|c|c|}
\hline & \multicolumn{6}{|c|}{ Plasma glucose categories (mmol/1) } \\
\hline & \multicolumn{3}{|c|}{ Fasting glucose criteria ${ }^{a}$} & \multicolumn{3}{|c|}{ 2-h glucose criteriab } \\
\hline CVD & $1.01(0.84-1.22)$ & $1.20(0.88-1.64)$ & $1.34(>0.10)$ & $1.32(1.12-1.56)$ & $1.40(1.02-1.92)$ & $12.09(<0.005)$ \\
\hline All-cause & $1.03(0.93-1.14)$ & $1.21(1.01-1.44)$ & $4.32(>0.10)$ & $1.37(1.25-1.51)$ & $1.73(1.45-2.06)$ & $61.35(<0.001)$ \\
\hline
\end{tabular}

a Using fasting plasma glucose $<6.1 \mathrm{mmol} / 1$ as reference group b Using 2-h post-load plasma glucose $<7.8 \mathrm{mmol} / \mathrm{l}$ as reference group

c Compared with the models with only the 2-h glucose criteria, $2 \mathrm{df}$

Table 2. Sex-specific estimated absolute number of excess deaths for subjects not diagnosed as previously having diabetes

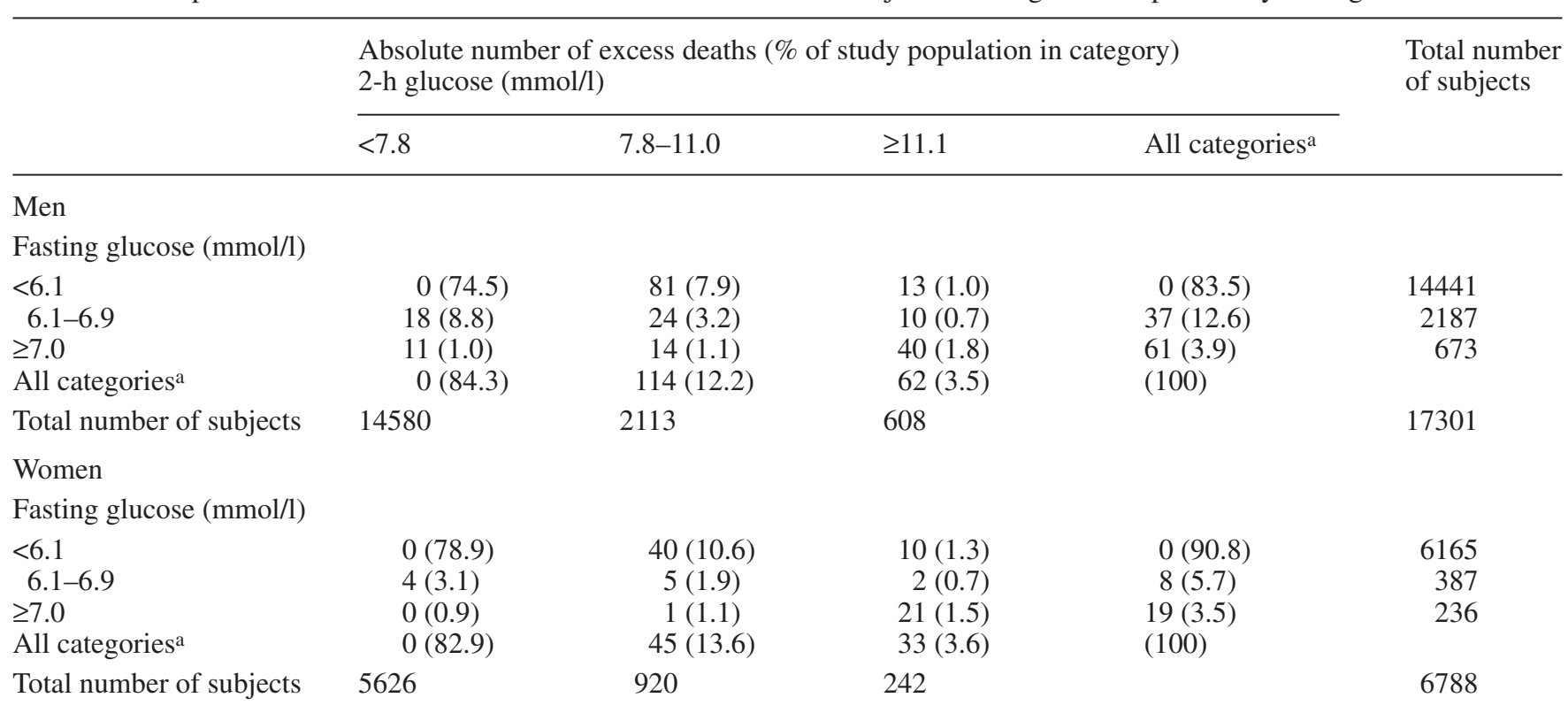

a Normoglycaemic category on fasting and 2-h glucose criteria is used as reference group for fasting and 2-h glucose categories; normoglycaemic category on fasting or 2-h glucose criteria alone is used separately for "All categories"

served in subjects with IGT especially those with IGT but normal fasting glucose concentrations [15, 16] (Table 2). This reflects the fact that IGT is the largest category of any abnormality in glucose homeostasis. In the DECODE study the prevalence of IGT was $13 \%$ [15] and among Asian populations this figure rose to $15 \%$ [17]. Compared with subjects with NGT, subjects with IGT had increased hazard ratios for mortality from all-cause mortality and CVD (Table 1). Thus, the large DECODE study confirmed that post-load hyperglycaemia increases mortality, and secondly, that post-load glucose is a better predictor of mortality than fasting glucose $[15,16]$. Further analyses of the DECODE data
dCompared with the models with only the fasting glucose criteria, $2 \mathrm{df}$

Adjusted for age, sex, center, total cholesterol, BMI, systolic blood pressure and smoking
Fasting and 2-h blood glucose categories are presented as plasma values

including $\mathrm{HbA}_{1 \mathrm{c}}$, show that 2-h blood glucose is a better predictor for all-cause mortality than either $\mathrm{HbA}_{1 \mathrm{c}}$ or fasting glucose concentrations [18]. Adding 2-h glucose to $\mathrm{HbA}_{1 \mathrm{c}}(p=0.001)$ or fasting glucose $(p=0.02)$ improved the prediction, whereas neither fasting glucose $(p=0.47)$ nor $\mathrm{HbA}_{1 \mathrm{c}}(p=0.42)$ added statistically significant information once 2 -h glucose was included in the model [18]. In a multivariate model including all three variables simultaneously, the hazards ratio $(95 \%$ CI) for a $1 \%$ increase in $\mathrm{HbA}_{1 \mathrm{c}}$ was 1.05 (0.87-1.26), for a $1 \mathrm{mmol} / \mathrm{l}$ increase in fasting glucose was 1.03 $(0.89-1.18)$ and for a $1 \mathrm{mmol} / \mathrm{l}$ increase in 2-h glucose was $1.06(1.01-1.11)$ [18]. 
The DIS identified mealtime hyperglycaemia to be an independent risk factor for myocardial infarction (MI) and all-cause mortality [19]. The detrimental effects of mealtime hyperglycaemia have also been linked directly to an increase in intima-media thickness (IMT), suggesting that mild-to-moderate mealtime hyperglycaemia is an independent risk factor for early atherosclerosis [20]. In a cohort at risk for diabetes, post-challenge glucose and glycaemic excursions were associated more strongly with carotid IMT than fasting glucose or $\mathrm{HbA}_{1 \mathrm{c}}$ concentrations [21].

Although there is much epidemiological evidence showing that post-load hyperglycaemia is an important risk predictor for future cardiovascular events, the question of whether this relation is causal is still open. The mechanism for the increased risk of mortality related to post-load hyperglycaemia has been speculated. Type 2 (non-insulin-dependent) diabetes mellitus is characterized by two fundamental defects: insufficient production of insulin by pancreatic beta cells and reduced target-tissue sensitivity to the effects of insulin (insulin resistance). An important defect is the impairment of early-phase insulin release during mealtime, which is always present in Type 2 diabetic patients and occurs early on in the development of the disease [22]. Early-phase insulin primes tissues that are sensitive to it, in particular the liver, which reduces hepatic glucose output. In subjects with IGT, the early-phase insulin response to glucose is reduced, and in Type 2 diabetic patients it is both delayed and blunted. The loss of early-phase insulin release has deleterious effects: hepatic glycogenolysis and gluconeogenesis are not inhibited sufficiently and glucose uptake by muscle is insufficient, leading to mealtime hyperglycaemia [23]. Postprandial hyperglycaemia could result in labile non-enzymatic glycation, promotion of thrombosis and in production of free radicals. These could cooperate in generating tissue damage and in favouring the development of micro and macro-complications [24]. Alternatively, the 2-h glucose concentration was directly related to the 2-h plasma insulin concentrations. Hyperinsulinaemia can result in body weight gain, accelerate the synthesis of very-low-density lipoprotein triglycerides, and decrease the concentration of high-density lipoprotein cholesterol contributing to a poor cardiovascular risk profile. Further negative effects include increased renal reabsorption of sodium, and an increase in coagulation profile (increased plasminogen activator-inhibitor-1) [23]. It is likely that postprandial hyperglycaemia is an independent risk factor for atherosclerosis and is also exerting an atherogenic effect indirectly through the clustering of a number of risk factors for atherosclerosis [23, 24].

There is accumulating evidence showing that deterioration of IGT to Type 2 diabetes can be prevented by lifestyle intervention [25, 26, 27, 28, 29]. We need to demonstrate that the prevention and control of mealtime hyperglycaemia will lead to a reduction of mortality, macrovascular disease and other late complications of Type 2 diabetes. In addition, there is a need to reconsider the thresholds used to diagnose hyperglycaemia. The majority of premature deaths due to hyperglycaemia occur in people with IGT $[15,16]$, therefore, it is important to pay medical attention to people whose 2-h post-challenge glucose concentration is within the IGT range. The first step is to detect such people through a systematic case finding among high risk groups, for example, those with a family history of diabetes or subjects with hypertension, obesity and previous gestational diabetes.

Acknowledgements. This paper is partly based on the data from the DECODE Study undertaken by the initiative of the European Diabetes Epidemiology Group. Investigators and study centers included in the DECODE Study are listed in references $[15,16]$. This work has been carried out with the help of a grant from Novartis Pharma AG, Basel, Switzerland.

\section{References}

1. International Collaborative Group (1979) Asymptomatic hyperglycemia and coronary heart disease: a series of papers by the International Collaborative Group based on studies in fifteen populations. J Chronic Dis 32: 683-837

2. Epstein FH (1985) Hyperglycemia as a risk factor for coronary heart disease. Monogr Atheroscler 13: 92-97

3. National Diabetes Data Group (1979) Classification and diagnosis of diabetes mellitus and other categories of glucose intolerance. Diabetes 28: 1039-1057

4. World Health Organization (1980) Diabetes Mellitus: WHO Expert Committee. WHO Technical Report Series, No. 646, second report. WHO, Geneva

5. World Health Organization (1985) Diabetes Mellitus: Report of a WHO Study Group. WHO Technical Report Series, No 727, WHO, Geneva

6. Rodriguez BL, Sharpp DS, Lau N et al. (1999) Glucose intolerance and 23-year risk of coronary heart disease and total mortality. Honolulu Heart Program. Diabetes Care 22: $1262-1265$

7. Tominaga M, Eguchi H, Manaka H, Igarashi K, Kato T, Sekikawa A (1999) Impaired glucose tolerance is a risk factor for cardiovascular disease, but not impaired fasting glucose. The Funagata Diabetes Study. Diabetes Care 22: 920-924

8. Vegt F de, Dekker JM, Ruhé HG et al. (1999) Hyperglycemia is associated with all-cause and cardiovascular mortality in the Hoorn population: the Hoorn Study. Diabetologia 42: 926-931

9. Balkau B, Shipley M, Jarrett RJ et al. (1998) High blood glucose concentration is a risk factor for mortality in middle-aged non-diabetic men. 20-year follow-up in the Whitehall Study, the Paris Prospective Study, and the Helsinki Policemen Study. Diabetes Care 21: 360-373

10. Shaw JE, Hodge AM, Courten $M$ de, Chitson P, Zimmet PZ (1999) Isolated post-challenge hyperglycemia confirmed as a risk factor for mortality. Diabetologia 42: $1050-1054$

11. Coutinho M, Gerstein HC, Wang Y, Yusuf S (1999) The relationship between glucose and incident cardiovascular events. A metaregression analysis of published data from 20 studies of 95,783 individuals followed for 12.4 years. Diabetes Care 22: 233-240 
12. Barrett-Connor E, Ferrara A (1998) Isolated post-challenge hyperglycemia and the risk of fatal CVD in older women and men. Diabetes Care 21: 1236-1239

13. Zimmet P (1979) Epidemiology of diabetes and its macrovascular complications in Pacific populations: The medical effects of social progress. Diabetes Care 2: 144-153

14. Guidelines Subcommittee (1999) World Health Organization-International Society of Hypertension Guidelines for the Management of Hypertension. J Hypertens 17: 151-183

15. DECODE Study Group (1999) Glucose tolerance and mortality: Comparison of WHO and American Diabetes Association diagnostic criteria. Lancet 354: 617-621

16. DECODE Study Group (2001) Glucose tolerance and cardiovascular mortality: comparison of the fasting and the 2-hour diagnostic criteria. Arch Intern Med 161: 397404

17. DECODA Study Group (2000) Comparison of the fasting and the 2-h glucose criteria for diabetes in different Asian cohorts. Diabetologia 43: 1470-1475

18. Qiao Q, de Vegt F, Nijpels G et al. (2000) 2h-BG is a better predictor for all-cause mortality than either $\mathrm{HbA}_{1 \mathrm{c}}$ or FPG. Diabetes Research and Clinical Practice, 17th International Diabetes Federation Abstract Book, Mexico City S129 (Abstract)

19. Hanefeld M, Fischer S, Julius U et al. (1996) The DIS Group. Risk factors for myocardial infarction and death in newly detected NIDDM: the Diabetes Intervention Study, 11-year follow-up. Diabetologia 39: 1577-1583

20. Hanefeld M, Koehler C, Schaper F, Fuecher K, Henkel E, Temelkova-Kurktschiev T (1999) Postprandial plasma glucose is an independent risk factor for increased carotid intima-media thickness in non-diabetic individuals. Atherosclerosis 144: 229-235
21. Temelkova-Kurktschiev T, Koehler C, Henkel E, Leonhardt W, Fuecher K, Hanefeld M (2000) Post-challenge plasma glucose and glycaemic spikes are more strongly associated with atherosclerosis than fasting glucose or HbA1c level. Diabetes Care 23: 1830-1834

22. Crepaldi G, Del Prata S (1995) What therapy do our NIDDM patients need? Insulin releasers. Diabetes Res Clin Pract 28 [Suppl]: S159-S165

23. Del Prato S (1999) Metabolic control in Type 2 diabetes: the impact of prandial glucose. Curr Opin Endocrinol Diabetes 6 [Suppl]: S1-S6

24. Ceriello A (1998) The emerging role of post-prandial hyperglycaemic spikes in the pathogenesis of diabetic complications. Diabet Med 15: 188-193

25. Eriksson KF, Lindgärde F (1991) Prevention of Type 2 (non-insulin-dependent) diabetes mellitus by diet and physical exercise. The 6-year Malmo feasibility study. Diabetologia 34: 891-898

26. Pan XR, Li GW, Hu YH et al. (1997) Effects of diet and exercise in preventing NIDDM in people with impaired glucose tolerance. The Da Qing IGT and Diabetes Study. Diabetes Care 20: 537-544

27. Eriksson J, Lindström J, Valle T et al. (1999) Prevention of Type II diabetes in subjects with impaired glucose tolerance: the Diabetes Prevention Study (DPS) in Finland. Diabetologia 42: 793-801

28. Tuomilehto J, Lindström J, Eriksson JG et al. (2001) Prevention of diabetes mellitus by lifestyle intervention in subjects with impaired glucose tolerance. New Engl J Med 344: $1343-1350$

29. Knowler WC, Barrett-Connor E, Fowler SE et al. (2002) Reduction in the incidence of type 2 diabetes with lifestyle intervention or metformin. N Engl J Med 346: 393-403 